\title{
Internet de las Cosas en Entornos Académicos. Caso de Éxito en la Universidad de Misiones
}

\author{
Milton E. Sosa, Eduardo O. Sosa \\ Secretaría de Investigación y Posgrado (SECIP) \\ Facultad de Ciencias Exactas Químicas y Naturales - Universidad Nacional de Misiones \\ Félix de Azara 1552 - N3300LQH Posadas - Argentina \\ \{mesosa,es\}@fceqyn.unam.edu.ar \\ Recibido: 10/11/16; Aceptado: 30/06/17
}

Resumen -- Actualmente, los equipos y artefactos destinados a la iluminación, ventilación y acondicionamiento del aire, en los ambientes de la Universidad de Misiones, se controlan de forma completamente manual. Se ha comprobado que dichos equipos $y$ artefactos permanecen conectados inclusive en ausencia de alumnos y docentes, no existiendo a la fecha control y actuación alguna sobre la temperatura ambiente y/o iluminación. El presente trabajo introduce el desarrollo inicial de un sistema de control automatizado inteligente de equipos $\mathbf{y}$ aparatos, morigerando así costos $y$ aportes al efecto invernadero. La solución planteada se lleva a cabo con ayuda de componentes económicos y usufructuando la infraestructura física existente en la dependencia. El sistema realiza el monitoreo, control de luminarias y de los equipos de acondicionamiento de aire, así como también, la presencia de personas en los ambientes. La central permite el accionamiento y control de los módulos en forma remota por medio de una aplicación desarrollada al efecto.

Abstract: Lighting, ventilation, and air conditioning environments at the Universidad Nacional de Misiones are variables manually controlled even today. It is a fact that devices remain connected even in the absence of students/teachers, with no control or capacity of implement some action on temperatures or artificial/natural lighting. It is necessary to start an intelligent automated control of equipment and appliances, to reduce substantially the energy cost and to contribute to cut down the greenhouse effect. The proposed solution is carried out by using non-expensive components and the existing physical infrastructure in the location. The system performs monitoring, control of lighting and air condition equipment, as well as the presence of people in the environments. The control unit allows driving and monitoring the built modules by an application developed for the purpose.

\section{Un díA DE Clases EN LA FACUlTAD}

Juan y Carla estudian Ingeniería Química en la Facultad de Ciencias Exactas, Químicas y Naturales (F.C.E.Q.y N.) de la Universidad Nacional de Misiones (UNaM). Los días viernes ambos deben asistir a clases en el módulo de la facultad construido en el Campus Universitario, conjuntamente con la cohorte a la que pertenecen. El Campus Universitario está situado aproximadamente a 8 kilómetros de los centros residenciales en la ciudad de Posadas. El día es nublado y caluroso, la temperatura primaveral alcanza los $31^{\circ} \mathrm{C}$. Los empleados administrativos entregan a los profesores de la clase las llaves de aulas habilitando su ingreso junto a los alumnos. En ese mismo momento encienden las luces del aula 7 donde se dictará clase ese día, y con el afán de mejorar las condiciones del aula se encienden los equipos de aire acondicionado (AA), los que se configuran de tal manera de mantener una temperatura en el aula de $24^{\circ} \mathrm{C}$. Considerando que el horario de trabajo del personal administrativo no coincide necesariamente con el de dictado de clases, el personal administrativo que cumple esas tareas se retira. Finalizada la clase, Juan y Carla se retiran a sus domicilios para así iniciar su merecido descanso del fin de semana, haciendo lo mismo los profesores.

El próximo día laborable al presentarse a cumplir sus funciones, probablemente el día lunes en la mejor de los casos, los administrativos comprueban que en el aula 7 la iluminación y los equipos de AA están aún funcionando.

La situación planteada es moneda común en la facultad, donde en algunos entornos en los cuales no existen equipos AA, existen ventiladores de techo, y en ciertos ambientes existen ambos elementos para confort de alumnos y profesores.

Considerando que el total de dependencias de la facultad incluye cerca de 75 aulas y laboratorios, sin considerar las dependencias administrativas, potencialmente la situación se podría reproducir 74 veces. Amén de los peligros que representa para cada uno de los entornos el funcionamiento de equipos sin supervisión y control, han transcurrido más de 60 horas, sin solución de continuidad, en los cuales se han estado dilapidando los exiguos recursos de las arcas de la Facultad de Ciencias Exactas Químicas y Naturales.

Lo expuesto hasta aquí, representando una situación cuasi absurda, demuestra la necesidad de establecer algún método inteligente de control y actuación sobre los elementos instalados en la Facultad.

\section{INTRODUCCIÓN}

La Internet de las Cosas (IoT, Internet of Things) representa un nuevo ambiente de cosas $\mathrm{u}$ objetos unívocamente identificables, los cuales pueden comunicarse entre ellos formando una red dinámica de alcance global [1]. La idea de IoT ha evolucionado y hoy en día las cosas no sólo se limitan a objetos identificables por RFID, sino que también involucran sensores, actuadores, o cualquier otro tipo de elemento, conectados a una red física y siendo identificables unívocamente.

Los entornos inteligentes representan escenarios avanzados de aplicación de las TIC's ${ }^{1}$, mediante las cuales interaccionan objetos de uso cotidiano en continua evolución como herramientas transparentes para usuarios no

${ }^{1}$ Tecnologías de la Información y Comunicación 
experimentados. Los entornos inteligentes han surgido rápidamente como un paradigma nuevo y emocionante que tiende a incluir diferentes campos de investigación de la computación ubicua y en red [2]. Podemos definir entonces a los ambientes inteligentes como "aquellas tecnologías significativas que se mimetizan entretejiéndose en la trama de la vida cotidiana hasta que se tornan indistinguibles de la vida misma" [3]. A partir del año 1999 se ha aplicado el término "Inteligencia Ambiental -AmI" [4] para describir una visión donde "las personas estarán rodeadas de interfaces inteligentes e intuitivas embebidas en objetos cotidianos de nuestro alrededor y un entorno que reconoce y responde a la presencia de individuos de manera invisible". Un entorno inteligente es el espacio integrador de un entorno físico con una colección de sistemas embebidos dedicados a la recopilación de información de un escenario determinado en un contexto heterogéneo [5] existiendo una infraestructura capaz de almacenar, compartir, entender y manejar esta información

En los edificios inteligentes se utilizan microcontroladores y sensores para automatizar diversos parámetros, entre ellos la iluminación y el acondicionamiento del aire, constatando la presencia de individuos tendiendo al ahorro energético y a una utilización más eficiente de la energía y al confort de los ocupantes de un ambiente. Domótica es un término de la tecnología de la información y la comunicación que se utiliza en edificios donde diversos componentes se comunican a través de la red local. Esta tecnología se puede aplicar para controlar, prevenir y actuar de acuerdo con los criterios seleccionados. Así cada uno de los elementos de la red es capaz de comunicarse con otras "cosas" del entorno a través de Internet, teléfonos fijos o teléfonos móviles. Un edificio inteligente es un tipo de edificio con tecnología instalada propia de los ambientes inteligentes [6].

La integración de las redes de sensores inalámbricos con la red cableada tradicional plantea diversos retos técnicos [7], centrados fundamentalmente en el desarrollo de sensores y de la infraestructura de redes de sensores (WSN).

En el contexto descripto, entre las cuestiones más difíciles de considerar se encuentran las de: ¿Cómo evaluar, construir y mantener un servicio en un ambiente inteligente de manera sustentable? ¿Cómo puede la sociedad donde habitamos disfrutar de éste pretendido estándar de vida elevado sin afectar a la naturaleza y el ambiente?

Para hallar respuestas válidas a las cuestiones precedentes, debemos hacer converger a los diferentes campos tecnológicos y sociales para que cooperen en el establecimiento del servicio pretendido. La evaluación de la sustentabilidad de las aplicaciones ha comenzado a ocupar un lugar preponderante en la concurrencia de mejorar la tecnología y reducir el consumo de recursos naturales existentes [8].

Las oficinas inteligentes surgieron como concepto durante la década pasada en Alemania. Gran parte de nuestra vida la pasamos en oficinas, por lo que éstas deberían ser sitios amigables que brindan un entorno agradable para sus usuarios. Pero, ¿Qué es una oficina inteligente? Se considera así a aquella que reconoce las actividades que se desarrollan en su entorno, siendo capaz de realizar un análisis de lo consumido, desperdiciado y gastado de los recursos disponibles. En tal sentido, debemos tener presente que los edificios, en forma global, son responsables del consumo de $17 \%$ del agua dulce, $15 \%$ de productos maderables, $33 \%$ de emisiones de $\mathrm{CO} 2$ y $40 \%$ de energía y materiales [9].

Se conocen como ambientes inteligentes a aquellas tecnologías que se presentan como sensibles, receptivas, adaptables, transparentes, omnipresentes e inteligentes [10]. Todos estos ambientes deben permitir la posibilidad de interacción entre los diferentes elementos que conforman los distintos entes. Se entiende por interoperabilidad [11], a la capacidad que poseen las entidades participantes en una comunicación de: 1) compartir cierto tipo de información, 2) procesar esa información de acuerdo a una semántica operacional previamente acordada.

Los hogares y oficinas inteligentes unifican el hecho de utilizar sensores y las tecnologías de la información de forma transparente para electrodomésticos y elementos de oficinas [12], tratando de administrar el uso eficiente de la energía a través de redes inteligentes. Esta es la base de construcción de Smart Grids, siendo éste tipo de redes de energía la fusión de redes eléctricas existentes y de los sistemas de comunicación para controlar los elementos de la red, beneficiando tanto a instalaciones residenciales como industriales y comerciales.

En el dominio emergente de las redes de sensores existe una amplia variedad de dispositivos con diferentes capacidades, desde sistemas relativamente potentes con procesadores de ordenadores y las interfaces inalámbricas de banda ancha -IEEE 802.11-, a otros de mucho menor capacidad, siendo los mismos nodos de baja potencia de cálculo, almacenamiento y transmisión. En este entorno es preciso contar indefectiblemente con herramientas de automatización avanzadas, las cuales deberán ser capaces de implementarse por medio de nóveles tecnologías de medición [13].

En este trabajo se considera que una arquitectura basada en múltiples agentes -que aplican inteligencia y colaboración para actuar sobre determinados elementos distribuidos definidos por el usuario- puede resolver procesos de decisión complejos, dinámicos, tal como los objetivos planteados en esta implementación de ambientes inteligentes.

El enfoque se fundamenta en el hecho que los acontecimientos internos del ambiente, generados ya sea por deseo de un usuario determinado o por una situación en el entorno; necesariamente deben ser controlados. Frente a un caso de incendio no se pretende activar simplemente una alarma. En tal caso, lo que se pretende -sobre la base de los servicios disponibles en términos de alarmas, rociadores, y puertas- es inferir el estado del ambiente y la ubicación de sus usuarios, para luego componer los servicios que garanticen la máxima seguridad para los alumnos o profesores presentes, así como también, la protección del edificio en sí mismo. Esta filosofía de diseño para entornos como los considerados en la UNaM, aportan también un valor agregado fundamental, y es que el sistema puede exportarse a instituciones similares con una reconfiguración mínima. De hecho, el mismo evento se tratará de manera diferente en diferentes lugares, 
simplemente porque los servicios disponibles serán distintos, como también lo será el ambiente.

\section{TRABAJOS RELACIONADOS}

Redes, sistemas y ambientes inteligentes son términos utilizados comúnmente por diversas comunidades, pudiendo encontrarse diferentes investigaciones aplicadas en distintos campos. En tal sentido, se pueden referir algunos trabajos relacionados a la construcción de ambientes residenciales y comerciales que se comportan inteligentemente ante diferentes situaciones propias de cada entorno. En su gran mayoría, tales trabajos refieren a ambientes domésticos -cf. [14], [15], [16], [17]. Por otra parte, y en menor media, otros trabajos refieren a edificios y oficinas -cf.: [18], [19], [20], [21]. Asimismo, la mayor parte de los trabajos tienden a ser bastante genéricos en la introducción de arquitecturas de alto nivel $\mathrm{y}$, por lo general, carecen de un reporte detallado de experimentación, considerando frecuentemente sólo una simulación del consumo de energía de los dispositivos.

El control a nivel de dispositivos se basa, generalmente, en trabajos de redes inteligentes considerando aspectos de precios, los cuales son un buen indicador de la disponibilidad de energía. En tales trabajos se consideran sistemas basados en agentes que negocian en un mercado virtual de administración de la energía [22] y, en algunos casos, haciendo uso también de estrategias propias derivadas de la Teoría de Juegos -cf.: [23], [24].

Por lo general, las acciones sobre los dispositivos reales en instalaciones propuestas solamente se simulan -cf.: [25], [26]- mientras que sólo unos pocos enfoques informan sobre el control realmente realizado a los ambientes, y las actuaciones realizadas en los mismos.

\section{MATERIALES, MÉTODOS E IMPLEMENTACIÓN}

\section{A. Infraestructura TI de la Facultad de Ciencias Exactas, Químicas y Naturales (FCEQyN)}

La infraestructura de tecnología de información -TI- de la FCEQyN comprende a los recursos de tecnología compartidos que proporcionan la plataforma para las aplicaciones de sistemas de información específicas de la Facultad. Dicha infraestructura incluye hardware y software que se comparte a través de toda la facultad; ergo son los elementos sobre los cuales se basan los diferentes servicios necesarios para mantener en funcionamiento todos los engranajes de la dependencia, tanto a nivel de docencia, de investigación o de gestión interna.

La red de datos de la facultad es, por necesidad, amplia y compleja. Esta red presenta distintos tipos de usuarios, tanto internos como externos, y llega a todas las dependencias de la facultad ofreciendo múltiples servicios; esto hace que se trabajen diversas subredes y cientos de nodos conectados a ella.

En las diversas dependencias de la facultad la infraestructura se basa en cableado estructurado, haciendo uso asimismo de accesos a redes inalámbricas sin controles centralizados de acceso tales como RADIUS o TACACS. Se conoce como cableado estructurado a la instalación integrada basada en estándares, de manera tal que se puedan prestar diversos servicios a través del mismo, por ejemplo, datos, voz, video y control, pudiendo prestarse entonces todos los servicios por un cableado en común. El sistema de cableado de la facultad, correctamente diseñado e instalado, provee a los usuarios de una infraestructura con un desempeño predecible y con una capacidad de expansión por crecimiento vegetativo en un periodo prolongado.

\section{B. Implementación de TIA/EIA-485}

Habida cuenta que existe una infraestructura de cableado estructurado que recorre los ambientes a ser controlados, por cuestiones económicas y de funcionalidad se ha seleccionado una alternativa que utilice medio de transporte soportado por el cableado estructurado, en éste caso EIA-485.

Está definido como un sistema en bus de transmisión multipunto diferencial, permitiendo transmitir a altas velocidades largas distancias a través de canales ruidosos, ya que reduce los ruidos que aparecen en los voltajes producidos en la línea de transmisión. El medio físico de transmisión es un par entrelazado que admite hasta 32 estaciones en 1 solo par, con una longitud máxima de 1.200 metros operando entre 300 y 19200 bps y la comunicación semiduplex (ver Figura 1). La transmisión diferencial permite una configuración multipunto, que al tratarse de un estándar abierto y común permite diversas configuraciones y usos.

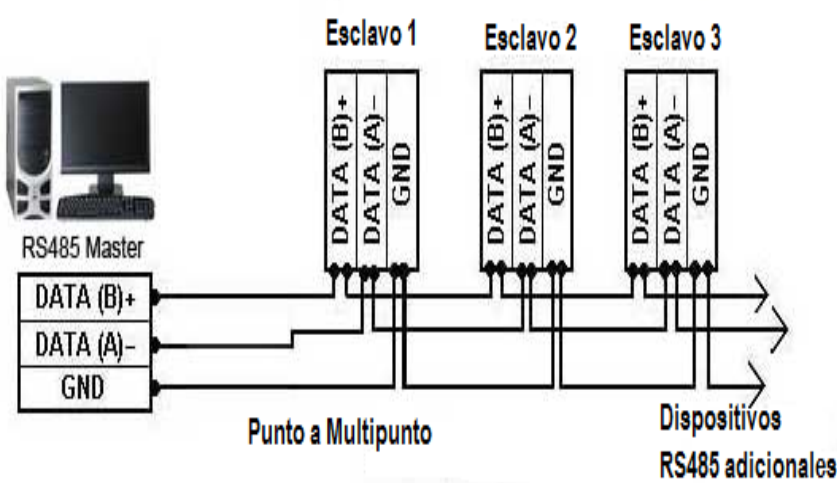

Fig 1. Bus de datos EIA RS-485

Las ventajas de esta norma para para su utilización con microcontroladores son su bajo costo, la capacidad de interconexión, la longitud de enlace y la tasa de transferencia [27].

\section{Hardware}

El hardware ideado, desarrollado e implementado forma parte de una red de topología bus (maestro/esclavo). Definida la convivencia de diferentes medios de comunicación, se ha desarrollado una adaptación híbrida; de esta manera la implementación permite comunicaciones por cables o inalámbrica.

El nodo maestro consta de una computadora de bajo costo Raspberry Pi [28] a la cual se adaptó un conector USBEIA/485 [29] desde el cual inicia el bus de comunicaciones RS-485 [30], realizando múltiples saltos de manera que cada nodo esclavo forme parte de la red. Este nodo se encuentra solidariamente configurado para formar parte de la red IPv4 de módulo Campus de la FCEQyN. Acceder desde el mundo exterior al nodo maestro para las tareas definidas ha requerido de apertura de ciertos puertos, y permisos por 
medio de ciertas reglas en diferentes cortafuegos de la facultad y de la Universidad.

Como escenario piloto en las dependencias se seleccionaron dos aulas modelo, las aulas 7 y 8 del anexo del campus de la FCEQyN. Cada uno de los nodos esclavo a instalar en las aulas ha sido desarrollado específicamente para ésta aplicación.

Cada nodo consta de un microcontrolador PIC 18 F2550 [31], el cual ha sido seleccionado debido a que su arquitectura de 8 bits es suficiente para desarrollar las operaciones que se requieren; y todas las herramientas necesarias, de programación como de compilación, son de libre y fácil acceso, asegurando de ésta manera una adecuada implementación libre de restricciones.

Todos los componentes de la implementación son controlados y accionados por el microcontrolador. Las tareas cumplidas por cada nodo son las siguientes:

a) Determinación de la temperatura ambiente por medio de un sensor de bajo costo LM35 [32].

b) Control de condición y estado de funcionamiento de los equipos de aire acondicionado, monitoreados a través de un sensor de falta de fase ubicado en bornes de la alimentación de cada equipo

c) Control por medio de contactores del tipo normal cerrado de la alimentación de la energía eléctrica a los acondicionadores de aire.

d) Monitoreo de presencia de personas en los diferentes ambientes

Para realizar el monitoreo de la presencia de individuos en los diferentes ambientes, el microcontrolador releva el estado de una señal proveniente de sensores de movimiento convencional, del tipo utilizado frecuentemente en sistemas de alarmas domiciliarias.

El nodo maestro se ha dispuesto físicamente en el rack de servidores y equipos del edificio. Adosado al tablero de energía se ha instalado el nodo actuador de corte de energía (Figura 2). Para que cada uno los nodos realicen la captura de datos, o realice alguna acción sobre los equipos y o artefactos involucrados, el nodo maestro debe enviar el comando específico.

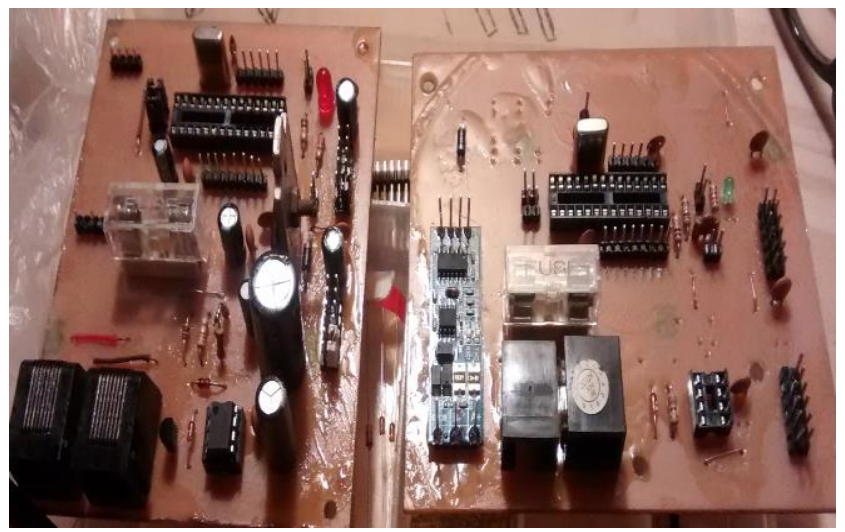

Fig. 2. Módulos desarrollados e implementados para tablero de control (izquierda) y diferentes aulas (derecha)

\section{Software}

En el nodo maestro se ha implementado LAMP ${ }^{2}$ definiendo la infraestructura de un servidor web, seleccionado por su bajo coste de adquisición y ubicuidad de sus componentes.

El software desarrollado e implementado en el nodo maestro realiza las siguientes tareas: a) consulta el estado de los diferentes nodos esclavos, b) registra los estados de los nodos en una base de datos MySQL c) presenta la información sobre los nodos esclavos al usuario final a través de una interfaz web (Figura 3), d) envía mensajes de alerta cuando alguno de los nodos falla, y e) interrumpe la alimentación de todos los nodos en caso de ser necesario

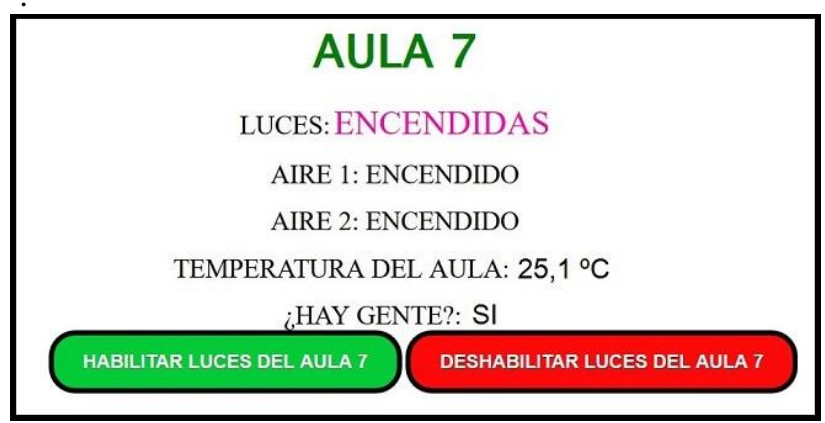

Fig 3. Informe web de variables controladas

Desde el nodo maestro se cumple con la función de monitorear el estado de las luminarias de las aulas y de los equipos AA, los cuales en caso de estar encendidos deben corresponderse con la presencia de individuos en los entornos bajo monitoreo. En caso de ausencia de notificación de presencia/movimiento, sumado a la temperatura del ambiente que es indicativa del funcionamiento de los equipos de aire acondicionado es posible interrumpir los circuitos de alimentación a los equipos. Como indicación de circuitos activos se ha instalado un sensor de falta de fase.

Si bien este sistema está diseñado para reaccionar de manera autónoma ante eventos que se pretenden controlar, se ha desarrollado una aplicación, la que siendo implementada en el nodo maestro, es accedida y controlada por medios de claves desde escenarios remotos, en el edificio central. La aplicación remite alertas por e-mail y Telegram [33] a la secretaría administrativa reportando algún tipo de evento (Figura 3).

Ante estas situaciones, es posible acceder por medio de protocolo HTTP al nodo maestro, donde se obtiene información en formato web los parámetros físicos tiempo real de las aulas. Considerando que las decisiones finales son tomadas por el personal destacado al efecto, es posible ejecutar acciones correctivas de manera remota. Ergo, es posible interrumpir la alimentación de energía tanto a los equipos de AA, o de alimentación de luminarias activando los contactores secundarios que son del tipo normal cerrado.

El normal funcionamiento de los contactores, ante cualquier inconveniente de falta de energía en la red de la proveedora, se ha asegurado alimentando las bobinas de los relés directamente desde los sistemas ininterrumpidos de energía (UPS) existentes en el edificio.

\footnotetext{
${ }^{2}$ Linux, Apache, MySQL y PHP-Python-Perl
} 


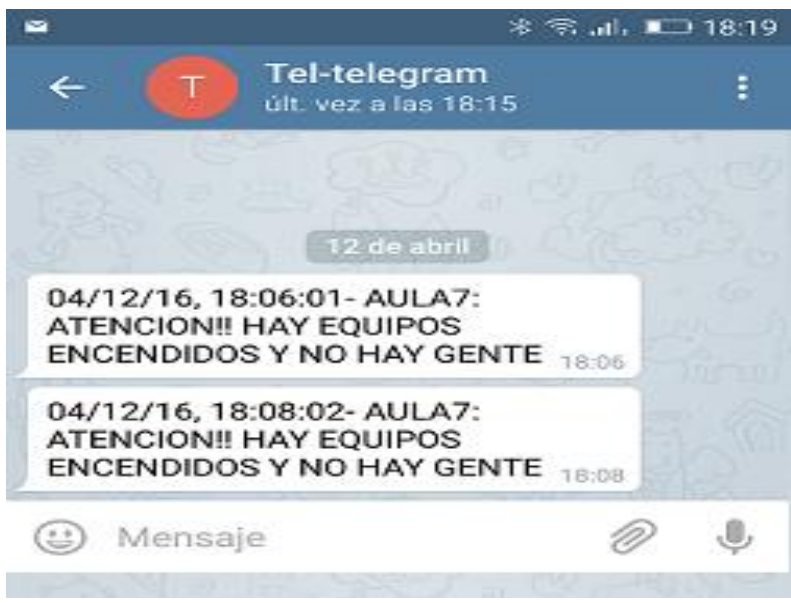

Fig 3. Reporte de variables por Telegram

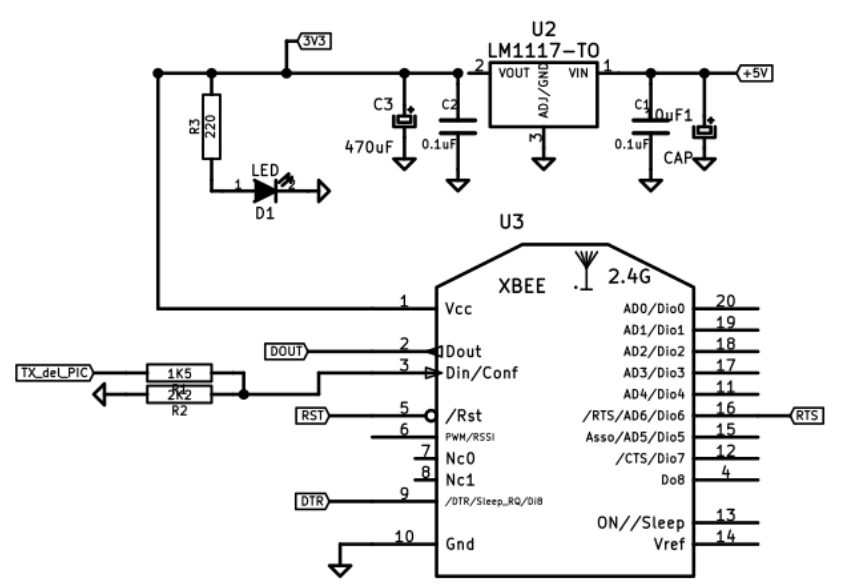

Fig. 4a.- Diagrama esquemático del adaptador de medios de comunicación

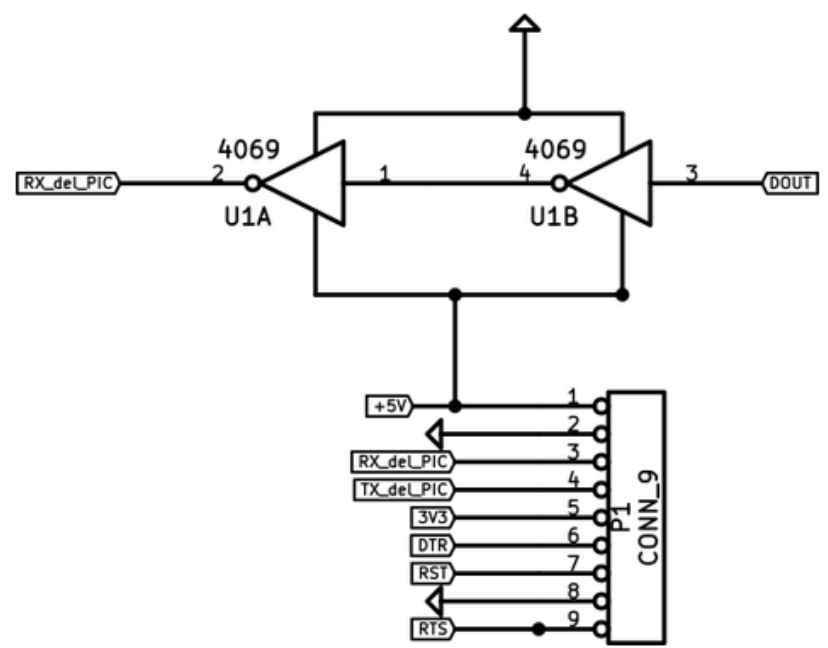

Fig. 4b.- Diagrama esquemático del adaptador de medios de comunicación

Si hipotéticamente, en un futuro próximo, se presenta la necesidad de una implementación híbrida - y para asegurar la escalabilidad, robustez y vida útil del sistema- se ha diseñado e implementado un nodo capaz de adaptar los niveles de señal desde el estándar EIA RS-485 (5 V) a la tensión necesaria en la lógica transistor-transistor (TTL) de 3,3 V, siendo ésta la tensión de operación los diferentes nodos disponibles en el mercado local. Este módulo no participa de forma activa de la red, ni generando información ni actuando de manera alguna; únicamente compatibiliza los medios de comunicación, siendo totalmente transparente para el medio cableado (RS-485) como para el medio inalámbrico (WSN). El diagrama esquemático se presenta en la figura 4a y 4b [34].

\section{CONClusiones y Trabajos Futuros}

La utilidad de Internet e IoT no es solamente encender las luces y desconectar los equipos causantes de despilfarro. La red llegará, en algún momento de su evolución, a ser indistinguible de los dispositivos que conecta; los cuales se convertirán en sí mismos en el ordenador.

Los rápidos avances de la microelectrónica y los transceptores de bajo costo han permitido nodos sensores con mayores rangos de lectura y tasas más elevadas de transferencia. La tecnología es ahora viable para nuevas aplicaciones novedosas con mayor movilidad y gran número de nodos. Sin embargo, a diferencia de los escenarios convencionales, estas nuevas aplicaciones requieren una plataforma de middleware más robusta y compleja con el fin de cubrir temas en diferentes capas de la arquitectura de comunicación, en diferentes contextos de negocios. Esta complejidad ha dejado varios temas de investigación abiertos en el diseño del middleware que significan aún un alto costo de ingreso en la adopción de la tecnología. De evolucionar la tecnología de manera esperada, existen dos grandes retos a fin de garantizar el acceso a la red sin fisuras; la coexistencia de las diferentes redes y el tamaño de IoT. Como trabajo futuro hemos considerado avanzar en la implementación de una red híbrida de manera de contribuir a tales puntos identificados como abiertos.

\section{REFERENCIAS}

[1] E. Borgia, «The Internet of Things vision: Key features, applications and open issues,» Computer Communications, vol. 54, pp. 1-31, 2014.

[2] E. Sosa, D. Godoy, R. Neis, G. Motta, R. Luft y D. Sosa, «Internet Del Futuro y Ciudades Inteligentes,» de Memorias Workshop de Investigadores en Ciencias de la Computación (WICC XV), Parana, 2013.

[3] M. Weiser, «The computer for the 21st century,» ACM SIGMOBILE Mobile Computing and Communications Review Special issue dedicated to Mark Weiser, vol. 3, no 3 , pp. 3-11, Jul 1999.

[4] Information Society Technologies Advisory Group, «Orientations for Workprogramme 2000 and beyond,» 1999. [En línea]. Available: http://ow.ly/uRncG.

[5] M. Sosa, L. Urbani, E. Sosa y D. Godoy, «Aportes a Smart Energy utilizando XBee e IEEE 802.15.4,» de Memorias del XVI Workshop de Investigadores en Ciencia de la Computacion (WICC), Ushuaia, 2014.

[6] H. Aspelund, T. Laberg y H. Thygesen, «Planning and management in municipal services", ».,» Norway Directorate for Social and Health, 1999.

[7] M. Gaynor, S. Moulton, M. Welsh, E. LaCombe, A. Rowan y J. Wynne, «Integrating wireless sensor networks with the grid,» Internet Computing, IEEE, vol. 8, nº 4, pp. 32-39, 2004.

[8] J. Kjeldskov, M. B. Skov, J. Paay y R. Pathmanathan, «Using mobile phones to support sustainability: a field study of residential electricity consumption,» de Proceedings of the SIGCHI Conference on Human Factors in Computing Systems, 2012.

[9] The World Green Building Council (WorldGBC), «Better places for People,» 12 Dec 2015. [En línea]. Available: http://www.worldgbc.org/. 
[10]D. Cook, J. Augusto y V. Jakkula, «Ambient intelligence: Technologies, applications, and opportunities,» Pervasive and Mobile Computing, vol. 5, $\mathrm{n}^{\circ}$ 4, pp. 277-298, 2009.

[11] L. Brownsword, D. Carney, D. Fisher, G. Lewis, E. Morris, P. Place, J. Smith, L. Wrage y B. Meyers, «Current Perspectives on Interoperability,» Pittsburgh, 2004.

[12] U. Premarathne, I. Khalil y M. Atiquzzaman, «Trust based reliable transmissions strategies for smart home energy consumption management in cognitive radio based smart grid, Ad $\mathrm{Hoc}$ Networks,» 2016

[13] R. J. Robles y T.-h. Kim, «Applications, Systems and Methods in Smart Home Technology: A Review,» International Journal of Advanced Science \& Technology, vol. 15, 2010.

[14] S. Tompros, N. Mouratidis, M. Draaijer, H. Foglar y H. Hrasnica, «Enabling applicability of energy saving applications on the appliances of the home environment,» IEEE Network, vol. 23, $\mathrm{n}^{\circ}$ 6, pp. 8-16, 2009.

[15] W. Lima, E. Souto, T. Rocha y R. a. P. Pazzi, «User activity recognition for energy saving in smart home environment,» de 2015 IEEE Symposium on Computers and Communication (ISCC), Larnaca, 2015.

[16] H. Lee, W. Park y J. Lee, «A Home Energy Management System for Energy-Efficient Smart Homes,» de 2014 International Conference on Computational Science and Computational Intelligence (CSCI), Las Vegas,, 2014.

[17] M. Chen y C. Lin, «Design and implementation of a smart home energy saving system by multi-microprocessors,» de 2015 IEEE International Conference on Consumer Electronics - Taiwan (ICCE-TW), Taipei, 2015.

[18] M. Choi, W. Park y I. Lee, «Smart Office Energy-Saving Service Using Bluetooth Low Energy Beacons and Smart Plugs,» de 2015 IEEE International Conference on Data Science and Data Intensive Systems, Sydney, 2015.

[19] R. Kusber, K. David y B. Klein, «A novel future Internet smart grid application for energy management in offices,» de Future Network and Mobile Summit (FutureNetworkSummit), Lisboa, 2013.

[20] C. Rottondi, M. Duchon, D. Koss, G. Verticale y B. Schätz, «An energy management system for a smart office environment,» de 2015 International Conference and Workshops on Networked Systems (NetSys), Cottbus, 2015.

[21] J. Pan, R. Jain, S. Paul, T. Vu, A. Saifullah y Sha.M., «An Internet of Things Framework for Smart Energy in Buildings: Designs, Prototype, and Experiments,» IEEE Internet of Things Journal, vol. $2, n^{\circ} 6$, pp. 527-537, 2015.

[22] E. Bompard y B. Han, «Market-Based Control in Emerging Distribution System Operation," in vol. 28, no. 4, pp. , Oct. 2013.,» IEEE Transactions on Power Delivery, vol. 28, n 4, pp. 2373 2382, 2013.

[23] X. Cai, C. Zhang, H. Yu, R. Bhar y B. Gooi, «Uncertainty aware minority game based energy management system for smart buildings,» de 2012 IEEE Innovative Smart Grid Technologies Asia (ISGT Asia), Tianjin, 2012.

[24] Q. La, W. Chan y B. Soong, «Power Management of Intelligent Buildings Facilitated by Smart Grid: A Market Approach,» IEEE Transactions on Smart Grid, vol. PP, n 99, pp. 1-12, 2015.

[25] R. Milliken, J. Cordwell, S. Anderson, R. Martin y D. Marshall, «Can smart devices assist in geometric model building?,",» de (SAS) Sensors Applications Symposium, Zadar, 2015.

[26] N. Cherifi, G. Grimaud, T. Vantroys y A. Boe, «Energy Consumption of Networked Embedded Systems,» de 3 International Conference on Future Internet of Things and Cloud (FiCloud), Roma, 2015.

[27] E. López Pérez, «Ingeniería en microcontroladores: Protocolo RS$485, » 2015$.

[28] Raspberry Pi Foundation, «Teach, Learn and Make with Raspberry $\mathrm{Pi}, \gg 15$ Oct 2015. [En línea]. Available: https://www.raspberrypi.org/.

[29] Future Technology Devices International Limited, «USB to RS485 Serial Converter Cable,» 27 May 2010. [En línea]. Available: http://bit.ly/1TeElC5. [Último acceso: 12 Febrero 2016].
[30] Maxim Integrated, «Guidelines for Proper Wiring of an RS-485 (TIA/EIA-485-A) Network,» 19 Nov 2001. [En línea]. Available: http://bit.ly/1L09YoK

[31] Microchip Technology Inc., «PIC18F2550 in Production,» 2016. [En línea]. Available: http://bit.ly/1RyVeBe.

[32] National Semiconductor Corporation, «LM35 Precision Centigrade Temperature Sensors,» Nov 2000. [En línea]. Available: http://bit.ly/1VL3YaW.

[33] Telegram, «Telegram, a new era of messaging,» 2016. [En línea]. Available: https://telegram.org/.

[34] M. E. Sosa, «Simple XBEE adapter with level shifters,» 15 Octubre 2015. [En línea]. Available: http://bit.ly/1Q3tC5I. 